\title{
Mucormycosis (The Black Fungus)
}

\author{
Khayria M Abdel-Gawad* \\ Department of Botany and Microbiology, Faculty of Science, Assiut University, Egypt \\ *Corresponding author: Khayria M Abdel-Gawad, Department of Botany and Microbiology, Faculty of Science, Assiut University, \\ Egypt.
}

To Cite This Article: Khayria M Abdel-Gawad. Mucormycosis (The Black Fungus). Am J Biomed Sci \& Res. 2021 - 13(4). AJBSR.MS.ID.001896. DOI: 10.34297/AJBSR.2021.13.001896.

Received: 䟧 July 07, 2021; Published: 㠿 July 22, 2021

\section{Introduction}

Mucormycosis (zygomycosis, phycomycosis) is referring to infections caused by diagonal of order Mucorales [1]. While the diagonal of this order usually attack persons susceptible to infection of the nasal mucosa and ketoacidosis, or in conditions of immunosuppression like diabetes, solid organ transplant, severe burns, etc. [1]. Although, fungal infectious agents causing minor or major orbital infections [2]. Also, Mucormycosis, affects the sinuses, the brain and the lungs, immunocompromised individuals, such as cancer patients or people with HIV/AIDS and can be life-threatening in diabetic patients [3]. In the point of view in India about 12,000 cases of black fungus have been recorded [4], most of them among patients recovering from Covid-19 [4]. While the risk of death from it is $50 \%$, but Mucormycosis infection is usually rare [4].

It is observed that, In May 2021, in India health experts believe that the reason for the increase in cases in this way is due to the high incidence of diabetes in Indian patients [5]. This is due to the persons exposure to mucor mold which is commonly found in soil, plants, manure, and decaying fruits and vegetables [6]. Doctors believe that the use of steroids, a life-saving treatment for severe and critically ill Covid-19 patients [3]. On the other hand, steroids reduce inflammation in the lungs for Covid-19 and also, appear to help stop some of the damage that can happen when the body's immune system goes into overdrive to fight off coronavirus. But they also reduce immunity and push up blood sugar levels in both diabetics and non-diabetic Covid-19 patients [3]. On the other hand, Mucormycosis causing symptoms like coughing out blood, blackening over the nose, blurred vision, chest pain, and breathing difficulties [7]. While a classic feature of this infection is tissue necrosis or tissue death. But, if the fungus spreads to the eyes, the patient may develop blurred vision, drooping eyelids, swelling, and possible vision loss [7].

\section{Phylum: Zygomycota}

The zygomycetes are facultative saprophytes. They are terrestrial but, some of them are parasites. The hyphae aseptate. But septa are found in the reproductive structures only [8]. Rhizoids and columella are also occurring.

\section{Rhizopus (Black Bread Mold)}

\section{Reproduction in Zygomycota}

\section{Asexual reproduction}

It takes place by vegetative methods and sporulation. Vegetative reproduction occures by fragmentation, chlamydospore and oidia formation, but it absent in Rhizopus stolonifer [9].

\section{Sporulation}

Comprises the formation of sporangiophores which bearing young sporangium. Arises from mycelia, the sporangium divided into the denser peripheral, sporiferous zone and central domeshaped zone, is the columella [9]. The sporangiospores inside the sporangia produce haploid spores by mitosis. The spores produce new mycelia [8].

\section{Sexual Reproduction}

May occur, when environmental conditions deteriorate.by conjugation type [9]. The two different opposite mating types (+strain hypha and - strain hypha) produce structures that contain several haploid nuclei (progametangium). When the gametangium 
are mature, the intervening fusion septum between them dissolves [9]. The fusing gametangium from of two opposite mating types results in a heterokaryotic zygosporangium. The combined protoplast in the prozygosporangium containing a number of diploid nuclei is called the zygospore [9]. A thick-walled develops that protects the zygospore until environmental conditions become favorable. When conditions are favorable, nuclear fusion (karyogamy) occurs within the zygospore producing diploid nuclei. This is followed by meiosis. The zygospore then germinates to produce a sporangium which releases haploid spores [8].

\section{Zygomycosis}

Zygomycosis is referring to infection caused by diagonal of order Mucorales commonly involving rhinocerebral structures and occasionally the lungs, gastrointestinal tract, skin and other organ system [1]. The zygomycosis are all human fungal infections. Saprophytic diagonal belonging to the genera Rhizopus, Absidia and Mucor [1], by condition that compromise the immune system, particularly diabetes leukemia, lymphomata, prolonged Steroid and antibiotic treatments, organ transplantation, uremia, malnutrition, and general debilitation [1] (Figure 1-7).

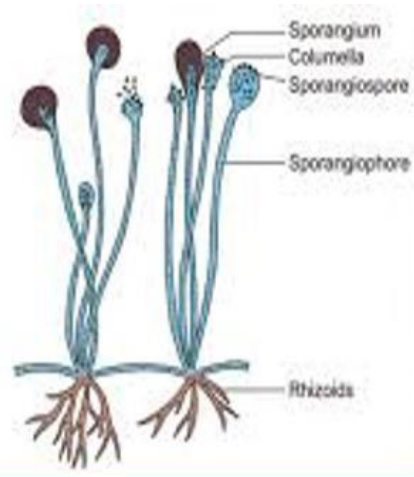

Rhizopus Morphology

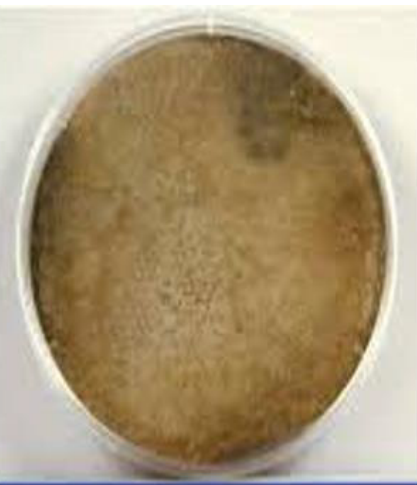

Rhizopus Growing on SDA

Figure 1.

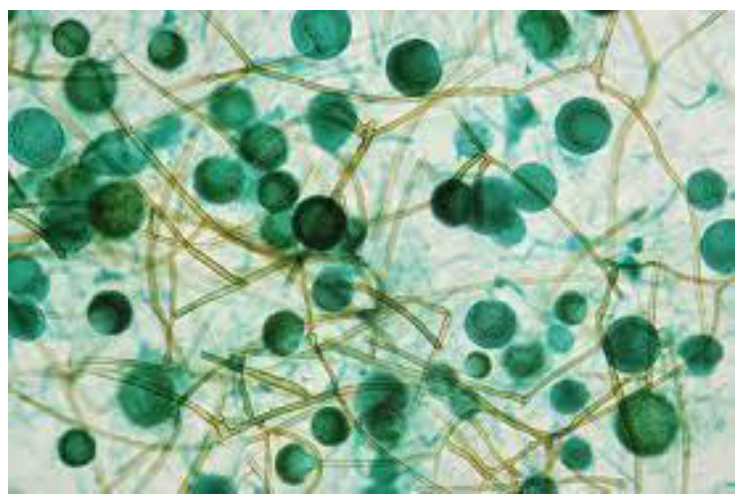

Figure 2.

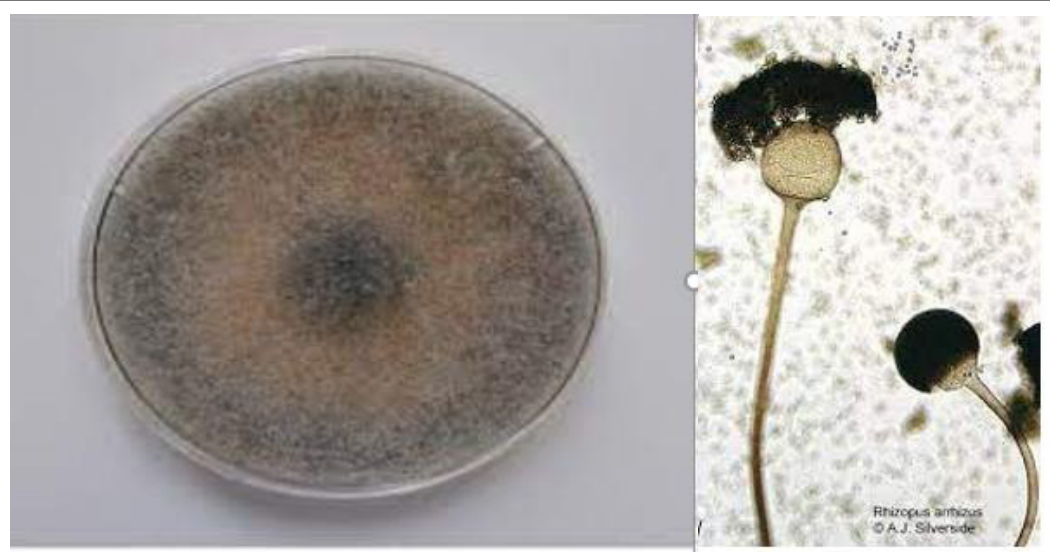

Figure 3: Rhizopus Arrhizus. 

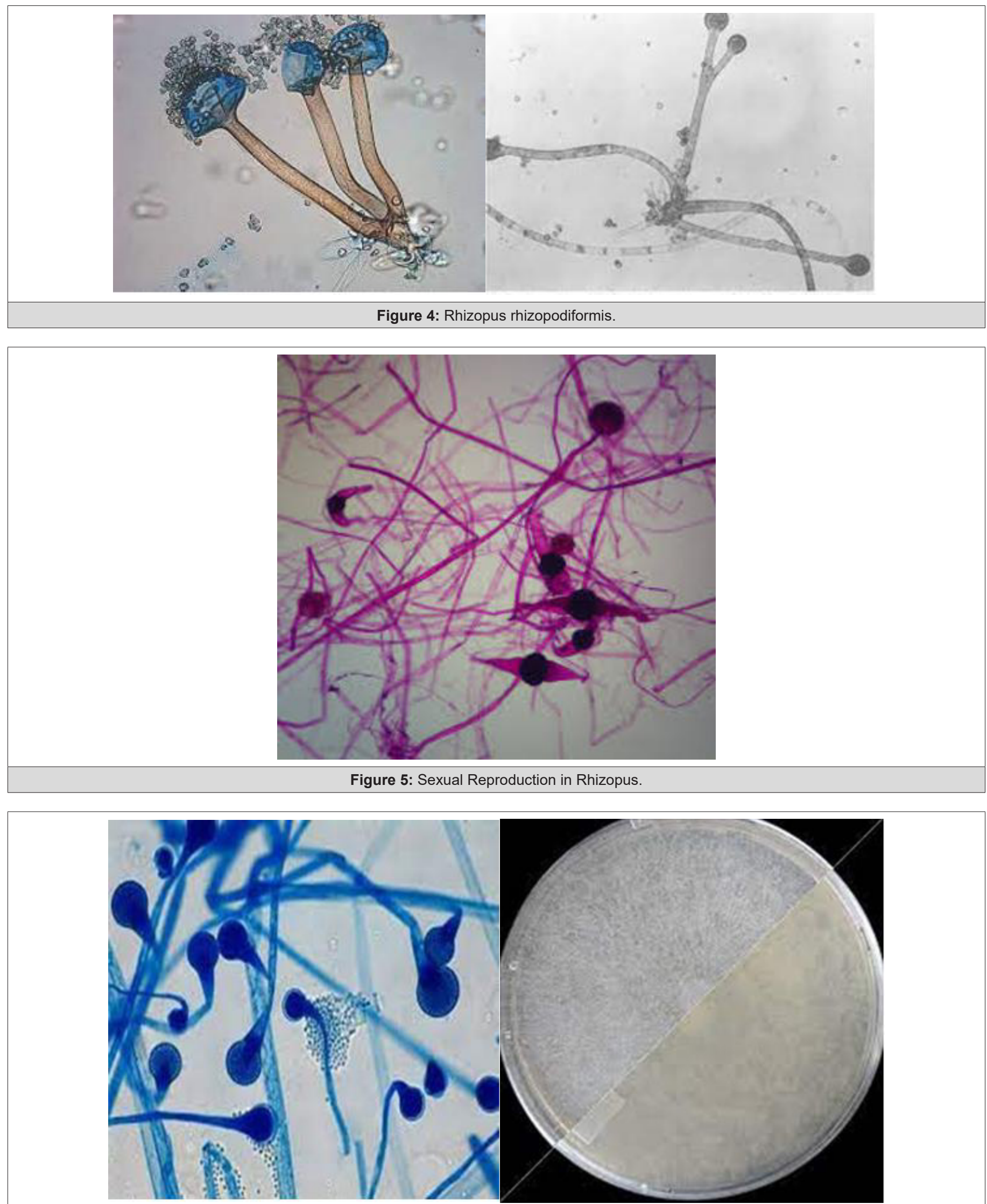

Figure 6: Absidia Corymbifera. 

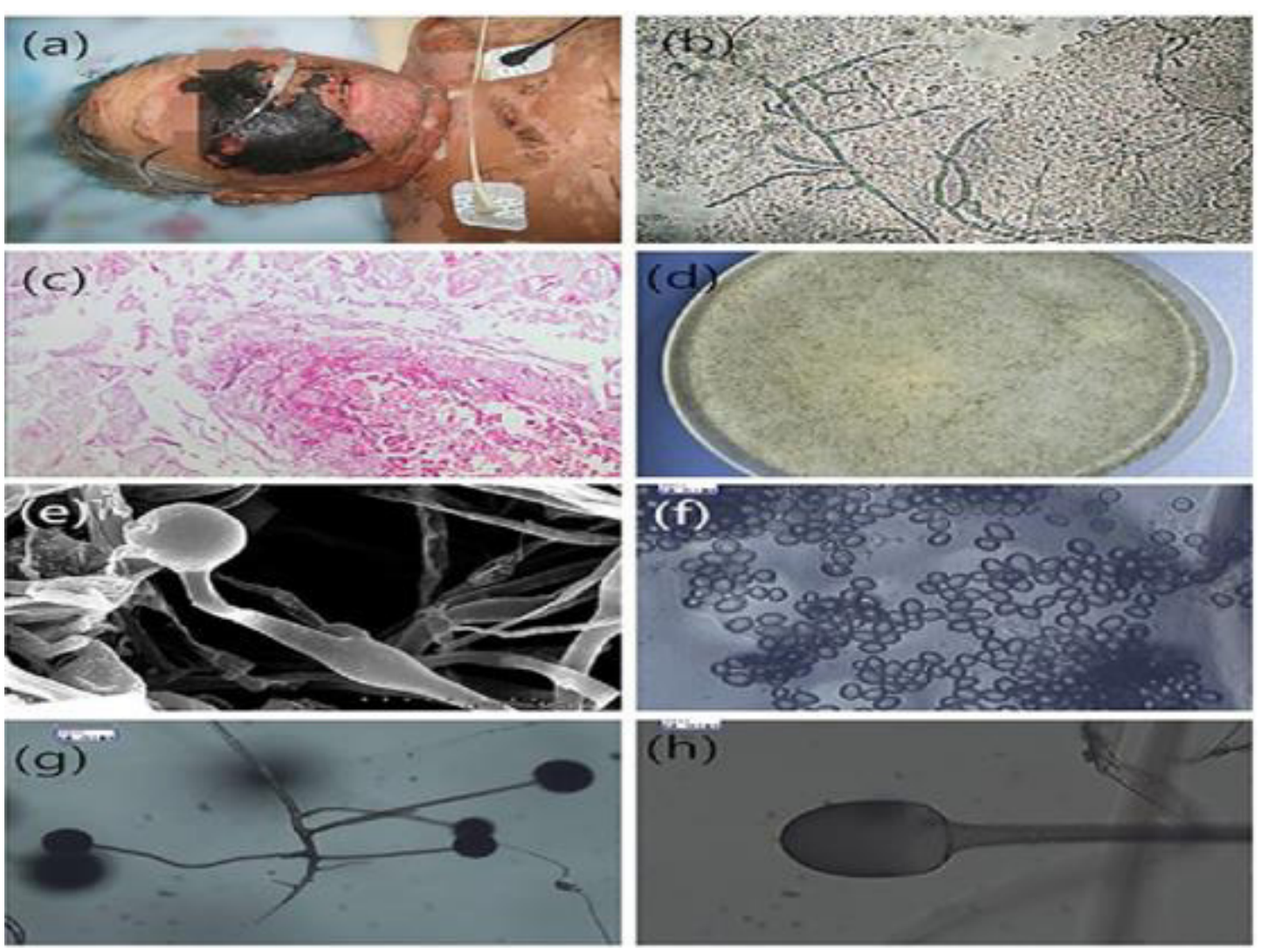

Figure 7.

\section{Rhinocerebral}

The Rhinocerebral zygomycosis occures association with diabetic ketoacidosis, but leukemic patients during periods of neutropenia are also susceptible [1]. The disease begins in the paranasal sinuses with fever headache or facial pain, black nasal discharge occures and extension to the hard palate or to perinasal skin, manifested by erythema at first and later by cyanosis and black necrotic eschar [1].

\section{Symptoms}

These include pain and redness around eyes and/or nose, fever, headache, coughing, shortness of breath, bloody vomits, and altered mental status. Warning signs can include toothache, loosening of teeth, blurred or double vision with pain [1].

\section{Gastrointestinal zygomycosis}

Most patients are children. The infection is associated with kwashiorkor, amebic dysentery, pellagra and typhoid [1].

\section{Symptoms}

Abdominal pain, atypical peptic ulcer symptoms, hematemesis, and hematochezia [1].

\section{Cutaneous Zygomycosis}

Primary mucorales infection the skin occure sporadically. Casses of infection in surgical wounds, insect bites, burns, and intravenous and intramuscular injection site. Infection occures in persons rendered susceptible by the forms of immunosuppression or debilitation in other fungal infection. Persons who develop zygomycotic infection under occlusive dressings respond readily to removal of the dressing and simple debridement [1].

\section{Preventions}

If you are visiting dusty construction sites, you can use masks. Also, you can wear shoes, long trousers, long-sleeved shirts and gloves while gardening. Maintain personal hygiene [10]. Also, use protective gloves and wash the hands always with soap and water.

\section{Conclusion}

From my point of view, every microbe has a dose to induce disease if a person is exposed to it. And COVID- 19 is the primary infectious pathogen that attacks the respiratory tract and the mucous membranes of the host nose and if the immune system is not overcomes on the disease, it reaches to the lungs. After the patient recovers from Corona, after high course of treatment by 
drugs ,then the affected tissues become exhausted, as well as the immunity becomes weak, especially in diabetic patients and this helps the secondary opportunistic weak infectious pathogen to grow and infect the same mucous tissues when the environment in which the patient is present is favorable for the growth of the fungus when high humidity and a temperature suitable for its growth, 28 or 37, and possible contamination of hospital equipment. The growth of order Mucorales fungal species is faster in penetrating into the tissues of the body, as it extends from the nose until it infects the eye and then extends to the brain. Its spores can travel in the bloodstream and infect other parts of the body.

\section{Treatment}

Once Mucormycosis is suspected, Amphotericin B [1] and [11], at an initial dose of $1 \mathrm{mg}$ is initially given slowly over 10-15 minutes into a vein, then given as a once daily dose according to body weight for the next 14 days. It may need to be continued for longer [5], and Isavuconazole and Posaconazole are alternatives also, used [11].

\section{References}

1. John Thorne Crissey, Heidi Lang and Lawrence Charles Parish (1955) Handbook Manual of Medical Mycology b Blackwell science p.1-263.
2. Santosh Kumar Swain, Smarita Lenka, Saurjya Ranjan Das (2020) Rhinoorbital Mucormycosis - A Dreaded Clinical Entity. International Journal of Current Research and Review.

3. Soutik Biswas (2021) Mucormycosis: The 'black fungus' maiming Covid patients in India. BBC News.

4. Shruti Menon (2021) Black fungus: Is diabetes behind India's high number of cases? BBC Reality Check.

5. Anuradha Mascarenhas | (2021) Mucormycosis or 'black fungus' in Covid-19 patients, its symptoms and treatment.

6. Tamil-Nadu News (2021) Should Tamil Nadu be worried about black fungus? 5 points. DT Next for the New Age Reader.

7. Angela Betsaida B Laguipo (2021) Black fungus complicates India's COVID pandemic. News Medical lifesciences.

8. Biology II Laboratory Manual (Lumen) (2021) Module 3 Fungi.

9. Vashishta BR (1995) Book in Botany for degree students: fungi Pp.518 9/e1995.

10. (2021) FPJ Web Desk: Mucormycosis How to prevent? When to suspect? Here is ICMR's detailed advisory.

11. Rajeev Soman, Ayesha Sunavala (2021) Post COVID-19 Mucormycosis from the Frying Pan into the Fire. J Assoc Physicians India 69(1): 13-14. 